\title{
Cuatro especies nuevas del grupo de especies Drosophila mesophragmatica (Diptera, Drosophilidae) de los Andes ecuatorianos
}

\author{
Diego Céspedes \& Violeta Rafael
}

Laboratorio de Genética Evolutiva, Escuela de Ciencias Biológicas, Pontificia Universidad Católica del Ecuador, Apartado: 17-01-2184, Quito, Ecuador. (diego_cesp@hotmail.com; vrafael@puce.edu.ec)

\begin{abstract}
Four new species of the Drosophila mesophragmatica species group (Diptera, Drosophilidae) from the Ecuadorian Andes. Four new species of Drosophila mesophragmatica group are described. The new species were found in paramo and remnants of Andean forest and in the Ecuadorian Andes. These species were captured from 2200 to $4000 \mathrm{~m}$. The new species are: D. cashapamba sp. nov., D. chorlavi sp. nov., D. rucux sp. nov. and D. yanayuyu sp. nov. With these new species, the number of species of the D. mesophragmatica group has been increase to 17. The species of this group are distributed mainly in the Andes of South America.
\end{abstract}

KEYWORDS. Diversity, genitalia, morphology, taxonomy.

RESUMEN. Se describen cuatro especies nuevas del grupo Drosophila mesophragmatica. Las nuevas especies fueron descubiertas en los páramos y remanentes de bosque andino de los Andes ecuatorianos. Las capturas fueron realizadas desde los 2200 hasta los $4000 \mathrm{~m}$ de altitud. Las especies nuevas son: D. cashapamba sp. nov., D. chorlavi sp. nov., D. rucux sp. nov. y D. yanayuyu sp. nov. Con las cuatro especies nuevas descubiertas, el número de especies dentro del grupo D. mesophragmatica se incrementa a 17 . Los miembros del grupo están distribuidos principalmente en los Andes sudamericanos.

PALABRAS-CLAVE. Diversidad, genitalia, morfología, taxonomía.

El género Drosophila Fallen, 1823 es sumamente diverso, con aproximadamente 1.146 especies descritas (BRAKe \& BäChli, 2008). Taxonómicamente, las especies de Drosophila han sido agrupadas en subgéneros y otros niveles infragenéricos como los grupos y subgrupos de especie que no son reconocidos por el Código Internacional de Nomenclatura Zoológica. En el Ecuador se han registrado cinco subgéneros, de los cuales Drosophila s. str. es el más diverso. Dentro de éste subgénero está el grupo mesophragmatica establecido por BRnCiC \& Koref-SANTIBAÑEZ (1957).

El grupo D. mesophragmatica comprende 13 especies (Vela \& Rafael, 2004): Drosophila canescens Duda, 1927; D. mesophragmatica Duda, 1927; D. gaucha Jaeger \& Salzano, 1953; D. altiplanica Brncic \& Koref-Santibañez, 1957; D. gasici Brncic, 1957; D. orkui Brncic \& Koref-Santibañez, 1957; D. pavani Brncic, 1957; D. viracochi Brncic \& Koref-Santibañez, 1957; D. camaronensis Brncic, 1957; D. brncici Hunter \& Hunter, 1964; D. amaguana Vela \& Rafael, 2004 D. ruminahui Vela \& Rafael, 2004 y D. shyri Vela \& Rafael, 2004.

Brncic \& Koref-Santibañez (1957) elaboraron una clave basada en características morfológicas externas que permite la identificación de tres categorías dentro del grupo D. mesophragmatica. La primera comprende una especie con cerdas escutelares anteriores convergentes (D. viracochi). La segunda está conformada por tres especies oscuras con cerdas escutelares anteriores divergentes (D. mesophragmatica, $D$. orkui y D. altiplanica) y la tercera integrada por dos especies de color café claro con cerdas escutelares anteriores divergentes (D. pavani y D. gaucha). Posteriormente NACRUR (1958) propuso la división de las seis especies del grupo D. mesophragmatica en dos linajes taxonómicos basados en la dirección de las cerdas escutelares anteriores. VELA \& RAFAEL (2004) reconocieron y actualizaron la propuesta, renombrando a los dos subgrupos como subgrupo $D$. viracochi que abarca las especies $D$. viracochi y D. ruminahui y el subgrupo $D$. mesophragmatica con las once especies restantes. Sin embargo, en un estudio filogenético de seis especies del grupo D. mesophragmatica utilizando marcadores nucleares y mitocondriales, Мота et al. (2008) establecieron tres subgrupos: subgrupo $D$. viracochi, integrado por $D$. viracochi; el subgrupo $D$. gaucha, formado por las especies hermanas $D$. pavani y $D$. gaucha y el subgrupo $D$. mesophragmatica, formado por D. gasici, D. brncici y D. mesophragmatica. Este estudio también concluye que $D$. viracochi sería la primera divergencia del grupo. Además, МотA et al. (2008) sugirieron que este y las demás separaciones habrían ocurrido en el Plioceno o Pleistoceno, posiblemente asociados con los refugios glaciares de los Andes.

En este trabajo se describen cuatro nuevas especies del grupo D. mesophragmatica capturadas en distintas localidades de los Andes ecuatorianos.

\section{MATERIALES Y MÉTODOS}

Se realizaron colectas en las provincias ecuatorianas de Pichincha e Imbabura desde el año 2004 hasta el 2010. Los individuos capturados fueron cultivados en medio de levadura, gelatina, limón y nipagina (RAFAel et al., 2000) a $18^{\circ} \mathrm{C}$. Con las hembras se fundaron isolineas y los machos fueron analizados e identificados mediante el examen de su genitalia. Para 
la obtención de las estructuras genitálicas se hirvió los tres últimos segmentos abdominales de las moscas en una solución de $\mathrm{KOH}$ al $10 \%$ durante 10 minutos. Luego se procedió a separar el arco genital, hipandrio y edeago en el caso de los machos y el ovipositor y las espermatecas en las hembras. Dichas estructuras fueron colocadas en placas excavadas y observadas en un microscopio compuesto para su respectiva identificación e ilustración. Los cuerpos fueron conservados en alcohol al 70\% y luego montados en alfileres entomológicos.

Como referencia para de los nombres de las estructuras se utilizó el libro de BächLı et al., (2004). Los dibujos fueron realizados utilizando el programa Adobe Illustrator ${ }^{\circledR}$ y las medidas de los individuos provenientes de isolineas fueron tomados en vivo en el programa AxioVision. Los holotipos, alotipos y paratipos fueron depositados en el Museo de Zoología sección Invertebrados de la Pontificia Universidad Católica del Ecuador, Quito (QCAZI).

\section{RESULTADOS}

\section{Drosophila cashapamba sp. nov. (Figs 1-7)}

Material tipo. Holotipo $\widehat{\partial}$ (disectado, genitalia en microtubo, montado en seco), Ecuador, Pichincha, Sangolquí (Cashapamba, $0^{\circ} 19^{\prime} 59,3^{\prime}$ 'S, 78²5'51'W, 2.417 m), VI. 2010, M. L. Figuero col., V. Rafael det. (QCAZI 2349); alotipo $\&$ (disectado, genitalia en microtubo, montado en seco), los mismos datos del holotipo, V. Rafael det. (QCAZI 2348); paratipos: $12 \hat{\jmath}$ y 7 ㅇ (disectado, genitalia en microtubo, montados en seco), Ecuador, VI. 2010, M. L. Figuero col., V. Rafael det. (QCAZI 2350-68).

Descripción del macho. Holotipo (en vivo, proviene de isolínea). Morfología externa: longitud total (cuerpo + alas) $5 \mathrm{~mm}$ y del cuerpo $3,5 \mathrm{~mm}$. Color del cuerpo marrón oscuro polinoso.

Cabeza. Primer y segundo segmento de las antenas color marrón oscuro y el tercer segmento de color marrón claro; arista plumosa, 4 ramas dorsales y 2 ventrales más la terminal bifurcada. Cabeza marrón claro ligeramente polinoso. La placa orbital color marrón; la cerda media más cerca de la orbital anterior, ligeramente hacia el borde exterior de la placa orbital. Triángulo ocelar y el triángulo frontal de color marrón oscuro. Frontal vitta de color marrón claro. Ocelos amarillos. Gena marrón claro con una cerda oral prominente. Carina prominente de color marrón claro ligeramente surcada. Ojos de color rojo vinoso. Palpos y proboscis de color amarillo.

Tórax. Color marrón ligeramente polinoso. Posee 8 hileras de pelos acrosticales entre las dorsocentrales anteriores. A lo largo del tórax, en dirección del punto de origen de las cerdas dorsocentrales, dos líneas delgadas y claras cuyos bordes son de color marrón oscuro. Escutelo marrón oscuro. Cerdas escutelares anteriores divergentes. La cerda esternopleural media es aproximadamente un cuarto de la longitud de la cerda anterior. Patas de color amarillo. Alas amarillentas con la celda costal y marginal ligeramente más oscuro, de $3,27 \mathrm{~mm}$ de largo y $1,41 \mathrm{~mm}$ de ancho. Índices alares: $\mathrm{c}=4,09 ; \mathrm{ac}=1,61 ; \mathrm{hb}=0,43 ; 4 \mathrm{c}=0,26 ; 4 \mathrm{v}=1,38 ; 5 \mathrm{x}=1,16$; $\mathrm{M}=0,38$; Prox. $\mathrm{x}=0,55$.

Abdomen. Color marrón amarillento, sin pigmentación oscura en los dos primeros segmentos, del tercer al quinto tergito presenta línea media dorsal y pigmentación oscura de forma triangular en la parte inferior de cada tergito que se va adelgazando hacia los lados hasta desaparecer.

Genitalia interna. En vista frontal el ápice del edeago es de forma triangular (Figs 1 - 3), termina en una punta bífida; los otros dos extremos son puntiagudos y quitinizados. Presenta abertura dorsal; apodema poco quitinizado, índice del edeago $=1,13$; las paráfisis son quitinizadas de forma rectangular, con una cerda en el extremo distal. Hipandrio en forma de V (Fig. 5), con el arco ligeramente quitinizado y la concha del hipandrio quitinizada y con una cerda en la parte baja e interna.

Genitalia externa. Placa anal parcialmente unida al epandrio (Fig. 4), sumamente pubescente y con cerdas muy largas. En la parte superior del epandrio presenta 4 cerdas, en su parte media 7 cerdas y en la parte inferior del arco 23 (20-24) cerdas. Surestilo de forma ovoide micropubescente; el lado derecho del surestilo presenta 10 dientes primarios (en la parte externa de los dientes primarios presenta microproyecciones), 8 (7-9) dientes secundarios en la parte superior y 8 (89) en la parte inferior además de 10 cerdas marginales y el lado izquierdo tiene 9 (7-9) dientes primarios (en la parte externa de los dientes primarios presenta microproyecciones), 5 (4-5) dientes secundarios en la parte superior y 7 (6-8) en la parte inferior, además de nueve cerdas marginales.

Descripción de la hembra. Alotipo (en vivo, proviene de isolínea). Morfología externa: presenta las mismas características del macho pero es ligeramente más grande.

Genitalia. Ovipositor quitinizado (Fig. 6), de color marrón; posee 4 dientes discales, 17 (15-18) cerdas marginales, una espina larga y otra pequeña y delgada. La espina larga está ubicada a nivel del cuarto diente discal. Espermateca pequeña no quitinizada (Fig. 7), transparente en forma de gorro y superficie irregular en la parte media superior. Índice de la espermateca $=1,98$ (relación base sobre altura).

Distribución. Esta especie ha sido colectada en bosque andinos cercanos varias localidades de la provincia de Pichincha (Cashapamba, $0^{\circ} 19^{\prime} 59,3$ "'S, 78 $25^{\prime} 51^{\prime \prime} \mathrm{W}$, $2.417 \mathrm{~m}$ e Intillacta $0^{\circ} 3^{\prime} 1,6^{\prime \prime} \mathrm{N}, 78^{\circ} 43^{\prime} 23,7^{\prime \prime} \mathrm{W}, 2.980$ m) y la Provincia de Santo Domingo de los Tsachillas (Guajalito, $0^{\circ} 13^{\prime} 48,8^{\prime}$ 'S, 7849'16,5”W, $2.980 \mathrm{~m}$ ).

Etimología. El nombre de la especie hace referencia al lugar de colecta, la Casa Hacienda Cashapamba, propiedad de la Pontificia Universidad Católica del Ecuador. 

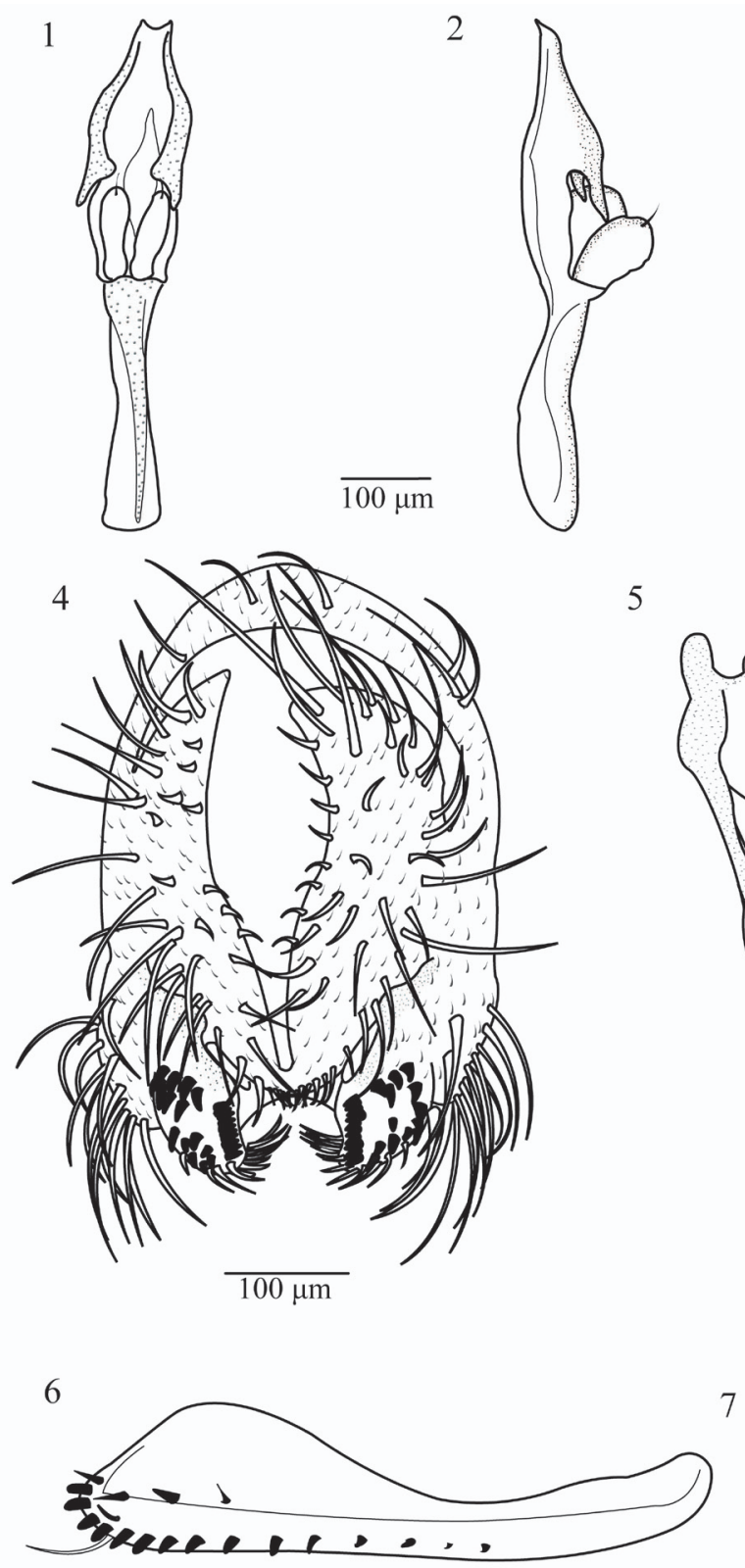

$100 \mu \mathrm{m}$

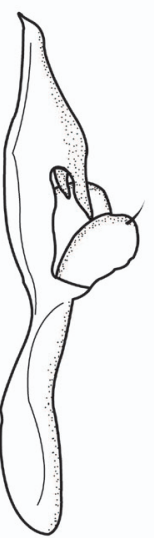

5

7
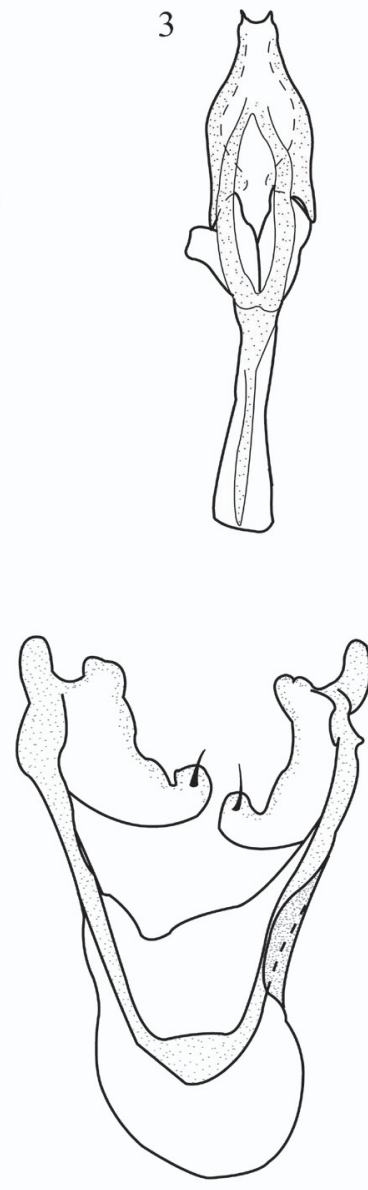

$100 \mu \mathrm{m}$

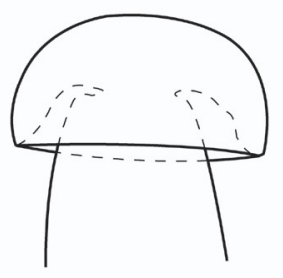

$50 \mu \mathrm{m}$

Figs 1-7. Drosophila cashapamba sp. nov., holotipo ^̊, edeago, apodema del edeago y paráfisis: 1, vista ventral; 2, vista lateral, 3, vista dorsal; 4, epandrio, cerci y surestilos; 5 , hipandrio y gonapófisis. Alotipo + : 6, ovipositor derecho, vista lateral; 7, espermateca.

\section{Drosophila chorlavi sp. nov.}

(Figs 8-14)

Material tipo. Holotipo $\widehat{\partial}$ (disectado, genitalia en microtubo, montado en seco), Ecuador, Imbabura, Ibarra (Chorlaví, 0²1’30”S, $78^{\circ} 10^{\prime} 42^{\prime \prime}$ W, 2.200 m), VI. 2004, M. L. Figuero col.,V. Rafael det. (QCAZI 2316); alotipo 9 (disectado, genitalia en microtubo, montado en seco), los mismos datos del holotipo, V. Rafael det. (QCAZI 2315); paratipos: 10 ก y 10 ㅇ (disectado, genitalia en microtubo), los mismos datos del holotipo, V. Rafael det. (QCAZI 2317-36).

Descripción del macho. Holotipo (en vivo, proviene de isolínea). Morfología externa: longitud total (cuerpo + alas) 3,5 $\mathrm{mm}$ y el cuerpo 2,4 $\mathrm{mm}$. Color del cuerpo marrón oscuro polinoso.
Cabeza. De color gris claro; arista plumosa, 4 ramas dorsales y 2 ventrales más la terminal bifurcada. Cabeza gris claro y de aspecto polinoso. Placa orbital estrecha y de color gris amarillento; cerda orbital anterior y posterior son de un tamaño similar, la cerda orbital media es la mitad del tamaño y se encuentra más cercana a la orbital anterior y ligeramente hacia el borde exterior de la placa orbital. Triángulos ocelar y frontal de color marrón oscuro. Frontal vitta de color marrón claro. Ocelos amarillos. Gena con una cerda oral prominente. Carina prominente ligeramente surcada, el surco mucho más oscuro que el resto de la cabeza. Ojos de color rojo vinoso. Palpos y proboscis amarillos, en el palpo se encuentran 3 cerdas bien desarrolladas. 
Tórax. Atigrado, mesonoto de color gris amarillento muy polinoso; las cerdas y pelos emergen de puntos oscuros. Posee 6 hileras de pelos acrosticales entre las dorsocentrales anteriores, siendo la última fila la más desarrollada. Escutelo polinoso y con una región más clara en la parte anterior. Cerdas escutelares anteriores convergentes. Cerda esternopleural media aproximadamente un cuarto de la longitud de la cerda anterior. Patas amarillas. Alas transparentes de 2,38 mm de largo y 1,04 $\mathrm{mm}$ de ancho. Índices alares: $\mathrm{c}=3,9$; $\mathrm{ac}=2,08 ; \mathrm{hb}=0,40 ; 4 \mathrm{c}=0,60 ; 4 \mathrm{v}=1,60 ; 5 \mathrm{x}=1,50 ; \mathrm{M}=0,60$; Prox $\mathrm{x}=0,80$.

Abdomen. Color amarillo y polinoso con línea media dorsal y una franja más oscura en la parte posterior de cada tergito, lateralmente todo el ancho del tergito es más oscuro.

Genitalia interna. En vista ventral el ápice del edeago es quitinizado, de forma triangular (Figs 8 10), termina en punta bífida. Presenta abertura dorsal y la parte distal se estrecha - y se continúa con un espacio en forma de pera. Apodema poco quitinizado, índice del edeago $=1,02$; las paráfisis tienen el borde quitinizado, son de forma rectangular y presenta una cerda de tamaño mediano en la parte distal además de 15 microproyecciones. Hipandrio en forma de V (Fig. 12), con la parte anterior membranosa y en la parte posterior, en la zona cercana a la concha del hipandrio, es membranosa y presenta numerosos microproyecciones. La concha del hipandrio de forma rectangular.

Genitalia externa. Cerci parcialmente unido al epandrio (Fig. 11). El epandrio en la parte superior presenta 4 cerdas, en su parte media 4 cerdas y en la parte inferior del arco 15 (13-19) cerdas. Surestilo de forma rectangular; presenta 11 dientes primarios y 10 (8-13) cerdas marginales. Decasternum rectangular.

Descripción de la hembra. Alotipo (en vivo, proviene de isolínea). Morfología externa: presenta las mismas características del macho pero es ligeramente más grande. El abdomen presenta las áreas laterales de los tergitos más oscuras que las del macho y ocupan un espacio más amplio.

Genitalia. Ovipositor quitinizado (Fig. 13), amarillo; posee 5 dientes discales, 14 (13-14) cerdas marginales, una espina larga y dos pequeñas y delgadas. La espina larga está ubicada a nivel del quinto diente discal. Espermateca quitinizada (Fig. 14), en forma de campana y superficie rugosa. Índice de la espermateca varía de 1 a 1,09.

Biología. Esta especie se puede mantener en laboratorio en el medio de cultivo de banano, levadura, gelatina, nipagina y limón (RAFAEL et al., 2000). Ocasionalmente ha sido capturada en frutos en descomposición de Cucurbita pepo (Cucurbitaceae) en la casa hacienda Cashapamba y en el Parque Arqueológico Rumipamba, Provincia de Pichincha, Ecuador.

Distribución. Esta especie ha sido colectada en las provincias ecuatorianas de Imbabura (Ibarra, Chorlaví) y Pichincha (Cashapamba, 0¹9'59,3”S, 78²5'51'W, 2.417 m, Quito, Parque Arqueologico Rumipamba, 00¹0'49,9'S, 78³0'04,8'W, 2.914 m y Loja, Izhcayluma, 04¹6'41,6”S, 79॰13'26,7’'W, $1.724 \mathrm{~m})$.

Etimología. El nombre de la especie hace referencia al lugar de colecta. Barrio Chorlaví de la ciudad de Ibarra, Provincia de Imbabura, Ecuador.

\section{Drosophila rucux sp. nov.}

(Figs 15 -21)

Material tipo. Holotipo $\widehat{\partial}$ (disectado, genitalia en microtubo, montado en seco), Ecuador, Pichincha, Quito (Cruz Loma, $0^{\circ} 1$ '19's, 78³1'25,1'W, 3.325 m), IV. 2008, D. Céspedes col., D. Céspedes det. (QCAZI 2305); alotipo + (disectado, genitalia en microtubo, montado en seco), IV. 2008, D. Céspedes col., D. Céspedes det. (QCAZI 2293); paratipos: $10 \hat{\text { y }} 10$ 오 (disectado, genitalia en microtubo, montado en seco), IV. 2008, D. Céspedes col., D. Céspedes det. (QCAZI 22942304; 2306-2314).

Descripción del macho. Holotipo (en vivo, proviene de isolínea). Morfología externa: longitud total (cuerpo + alas) $5 \mathrm{~mm}$ y solo el cuerpo 3,5 mm. Color del cuerpo marrón oscuro polinoso.

Cabeza. Primer y segundo segmento de las antenas color marrón claro; arista plumosa, 4 ramas dorsales y 3 ventrales más la terminal bifurcada (lado derecho roto). Cabeza marrón claro de aspecto polinoso. La placa orbital color marrón oscura; la cerda orbital anterior y posterior están hacia la parte interna de la placa orbital y la media ligeramente hacia el borde exterior de la placa orbital y ubicada más cerca de la cerda orbital anterior. Triángulo ocelar y el triángulo frontal de color marrón oscuro. Frontal vitta ligeramente más claro. Una cerda oral prominente. Carina prominente ligeramente surcada de color marrón claro. Ojos de color rojo vinoso. Palpos amarillos.

Tórax. Color marrón oscuro polinoso. Posee 6 hileras de pelos acrosticales entre las dorsocentrales anteriores. La última fila de pelos acrosticales más desarrolladas. Delante de las cerdas dorsocentrales anteriores se encuentran un par de pelos ligeramente más gruesos y largos que el resto de los pelos acrosticales, dando la apariencia de una tercera cerda dorsocentral. Ésta característica se presenta en la mayoría de los individuos pero en algunos la cerda es más notoria y está presente solo a un lado del tórax. En la parte central y a lo largo del tórax se observa una franja más obscura; en base a esta franja se ha logrado encontrar cuatro morfotipos: el primer morfotipo presenta una franja que cubre el espacio comprendido entre las cerdas dorsocentrales y llega hasta el escutelo, el segundo posee una línea delgada en el centro en el tórax que cubre dos filas de pelos acrosticales, el tercer presenta una franja delgada hasta la altura de las cerdas dorsocentrales anteriores que se ensancha hasta cubrir el escutelo y el cuarto no presenta ninguna pigmentación entre las cerdas dorsocentrales. Del lado externo de las cerdas dorsocentrales y a lo largo de todo el tórax se encuentra una línea ligeramente más clara. Escutelo marrón oscuro y los bordes de color marrón amarillento. 

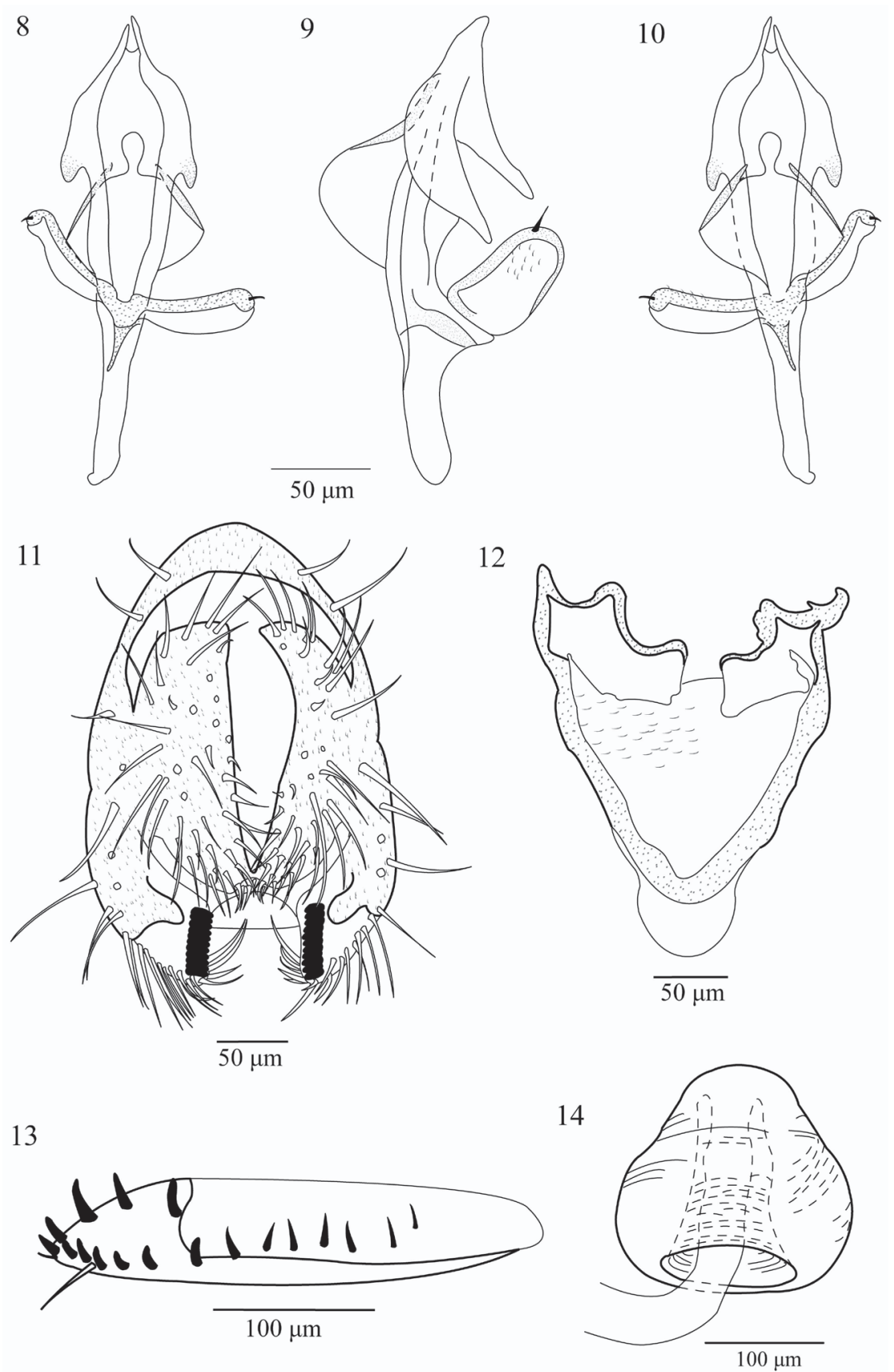

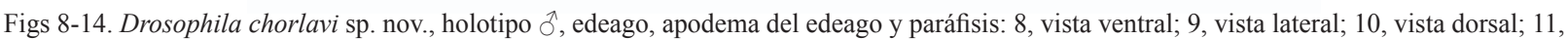
epandrio, cerci y surestilos; 12, hipandrio y gonapófisis. Alotipo $\circ: 13$, ovipositor derecho, vista lateral; 14, espermateca.

Cerdas escutelares anteriores divergentes. La cerda esternopleural media es aproximadamente un tercio de la longitud de la cerda anterior. Patas amarillas, los dos últimos segmentos tarsales de color marrón oscuro. Alas de color gris claro, de $3,64 \mathrm{~mm}$ de largo y 1,67 $\mathrm{mm}$ de ancho. Índices alares: $\mathrm{c}=3,80 ; \mathrm{ac}=2,00 ; \mathrm{hb}=0,22$; $4 \mathrm{c}=0,66 ; 4 \mathrm{v}=1,44 ; 5 \mathrm{x}=1,09 ; \mathrm{M}=0,44$; Prox. $\mathrm{x}=0,74$.

Abdomen. Color amarillo con línea media dorsal, la parte inferior de cada tergito es oscura y los márgenes laterales son totalmente oscuros. Testículos de color amarillo intenso (distinguibles a través del abdomen).

Genitalia interna. Ápice del edeago quitinizado y ensanchado en la parte media (Figs 15 - 17); la parte superior es angosta y termina en una punta curva dirigida hacia la parte dorsal; apodema poco quitinizado, índice del edeago $=2,16$; las paráfisis son quitinizadas, desnudas pero en algunos individuos se presentan de una a 7 microproyecciones en una o ambas paráfisis. Estas paráfisis son alargadas llegando a alcanzar las dos terceras partes del ápice del edeago y presenta en el extremo distal una cerda larga. Hipandrio tan largo como el arco genital (Fig. 19). Testículos con 3,5 vueltas externas y 2,5 vueltas internas.

Genitalia externa. Cerci parcialmente unida al epandrio (Fig. 18). Epandrio y surestilo micropubescentes; el lado derecho del surestilo presenta 12 dientes primarios, 
con 16 (15-19) cerdas marginales y el lado izquierdo 10 dientes primarios (en el holotipo separados por un espacio que correspondería a 2 dientes ausentes) con 16 (14-19) cerdas marginales.

Descripción de la hembra. Alotipo (en vivo, proviene de isolínea). Morfología externa: presenta las mismas características del macho pero es ligeramente más clara.

Genitalia. Ovipositor muy quitinizado (Fig. 20), de color marrón; posee 5 dientes discales, 13 (13-14) cerdas marginales, una espina larga y otra pequeña y delgada. La espina larga está ubicada entre el segundo y tercer diente discal. Espermateca pequeña no quitinizada
(Fig. 21), transparente (algunos individuos ligeramente marrón) en forma de gorro, superficie irregular en la parte media superior. Índice de la espermateca $=0,88$ (relación base sobre altura).

Distribución. Esta especie también ha sido capturada en las localidades de Antisanillo ( $0^{\circ} 24^{\prime} 33,3^{\prime \prime} \mathrm{S}$, $\left.78^{\circ} 12^{\prime} 28,4^{\prime \prime} \mathrm{W}, 3.352 \mathrm{~m}\right)$ y Peñas Blancas, vía Pifo, Papallacta $\left(0^{\circ} 17^{\prime} 56,1^{\prime \prime} \mathrm{S}, \quad 78^{\circ} 14^{\prime} 37,5^{\prime \prime} \mathrm{W}, 3.628 \mathrm{~m}\right)$, Provincia de Pichincha, Ecuador.

Etimología. D. rucux fue capturada en las estribaciones del Volcán Rucu Pichincha; Pichincha, Ecuador. Rucu es una palabra que proviene del idioma quichua y significa viejo.
15
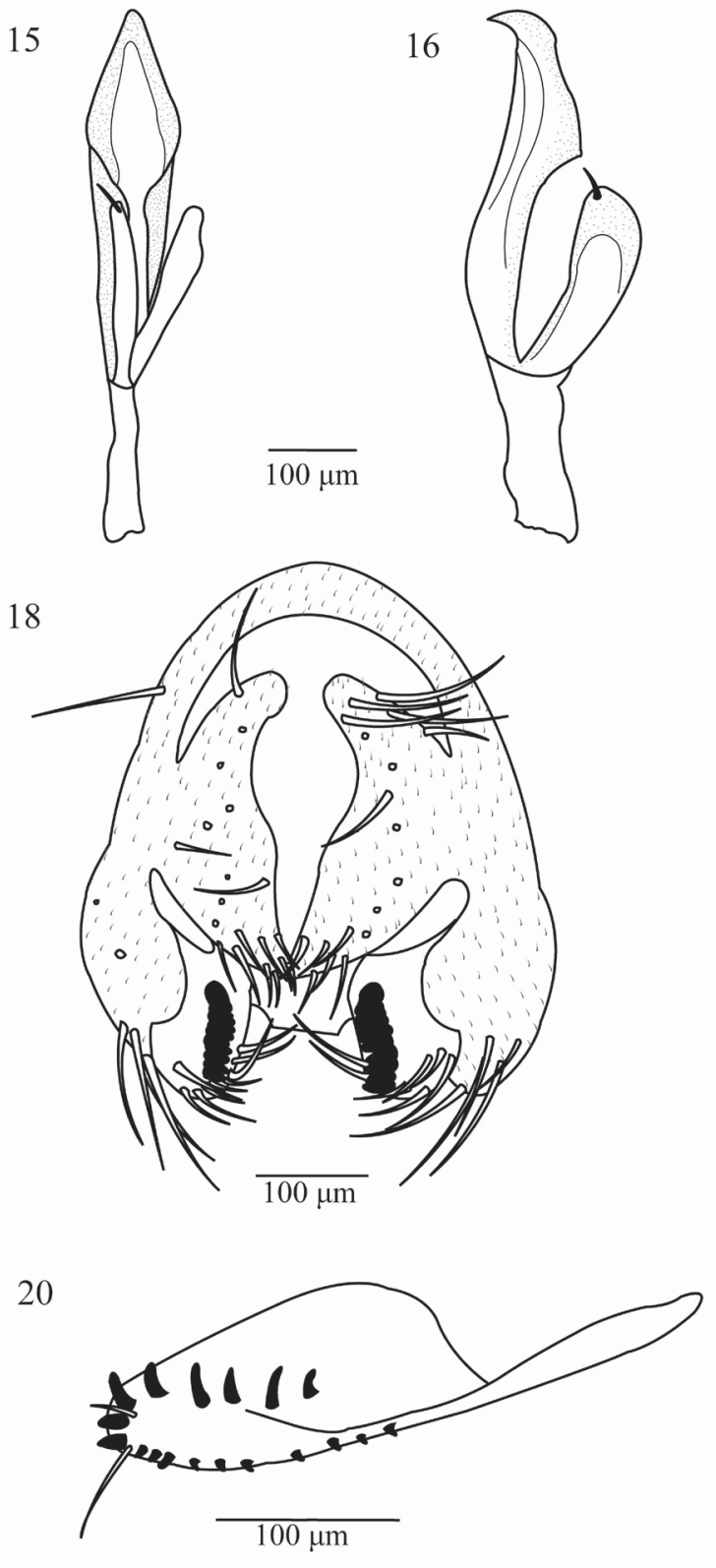

20

18
16

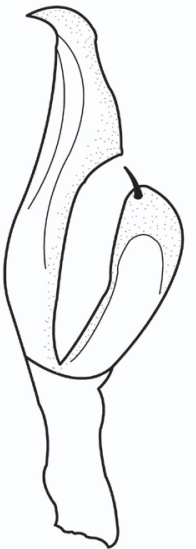

17

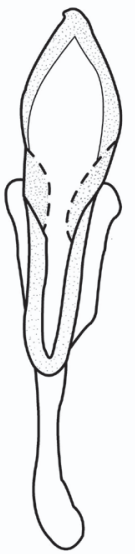

19

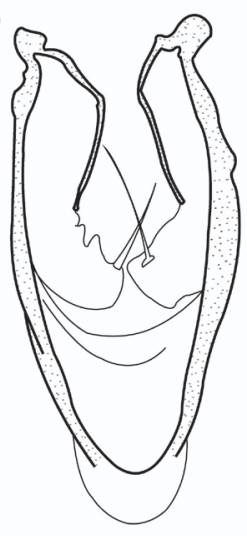

$100 \mu \mathrm{m}$

21

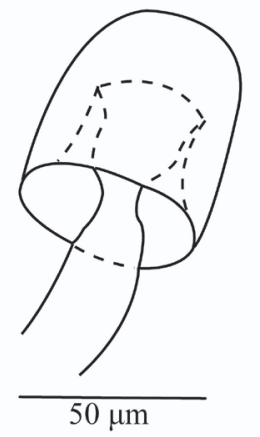

Figs 15-21. Drosophila rucux sp. nov., holotipo ổ, edeago, apodema del edeago y paráfisis: 15, vista ventral; 16, vista lateral; 17, vista dorsal; 18 , epandrio, cerci y surestilos; 19, hipandrio y gonapófisis. Alotipo + : 20, ovipositor derecho, vista lateral; 21, espermateca. 
22

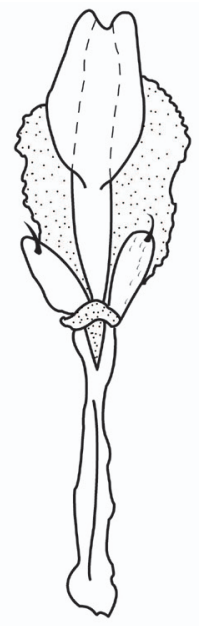

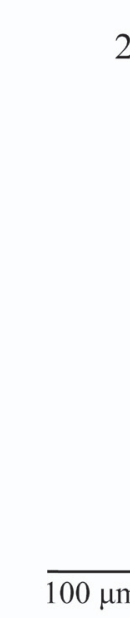

23

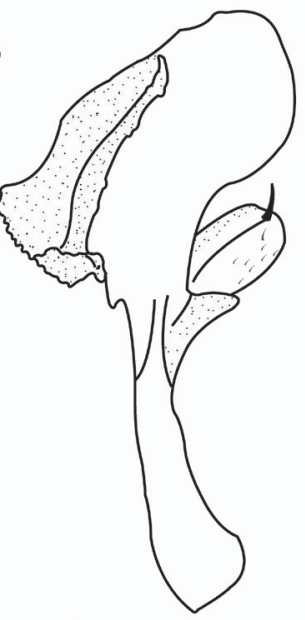

24

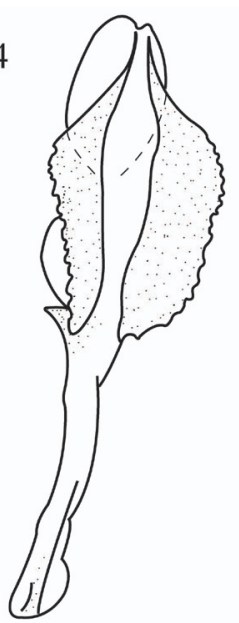

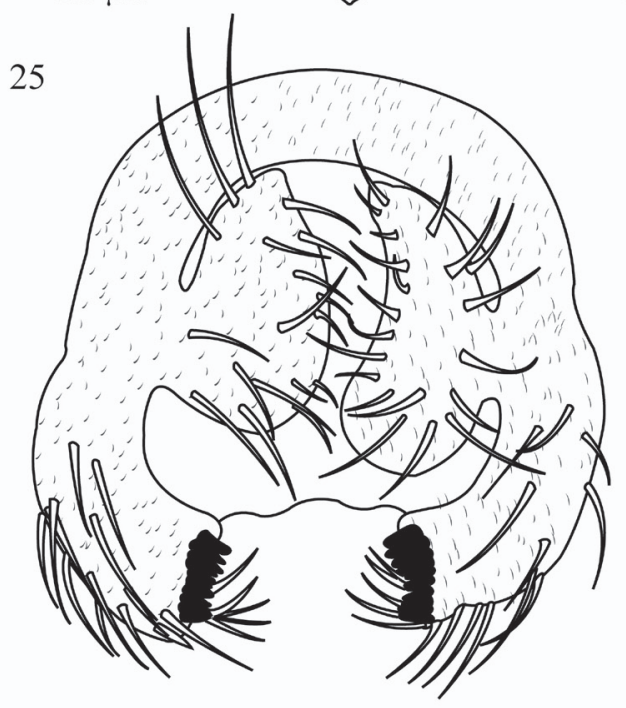

$100 \mu \mathrm{m}$
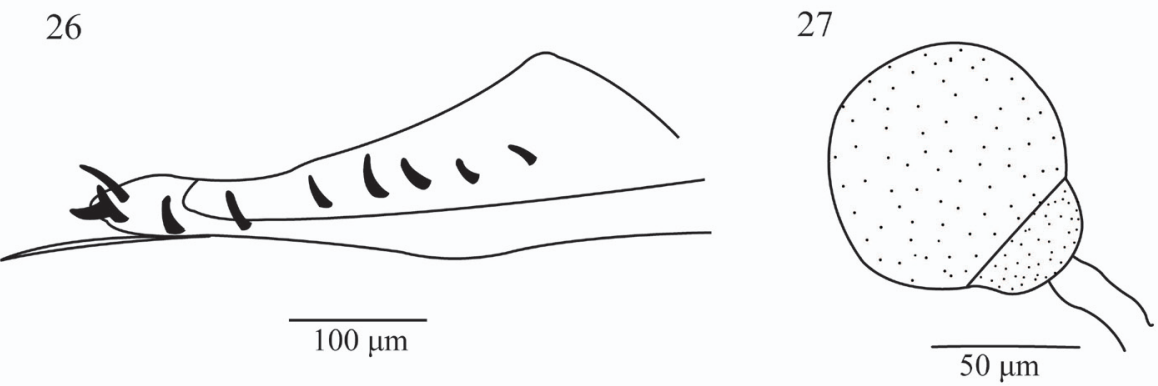

Figs 22-27. Drosophila yanayuyu sp. nov., holotipo ổ, edeago, apodema del edeago y paráfisis: 22, vista ventral; 23, vista lateral; 24, vista dorsal; 25 , epandrio, cerci y surestilos. Alotipo + : 26, ovipositor derecho vista lateral; 27, espermateca.

\section{Drosophila yanayuyu sp. nov.}

(Figs 22-27)

Material tipo. Holotipo $\widehat{o}$ (disectado, genitalia en microtubo, montado en seco), Ecuador, Pichincha, Quito (Cruz Loma, $0^{\circ} 1$ '19'S, 78³1'25,1’W, 3.325 m), IV. 2008, D. Céspedes col., D. Céspedes det. (QCAZI 2383); alotipo 9 (disectado, genitalia en microtubo, montado en seco), IV. 2008, D. Céspedes col., D. Céspedes det. (QCAZI 2284); 3 paratipos ( (disectado, genitalia en microtubo), IV. 2008, D. Céspedes col., D. Céspedes det. (QCAZI 2285-87).

Descripción del macho. Holotipo (proviene de isolínea). Morfología externa: longitud total (cuerpo + alas) 4,70 mm y solo el cuerpo $3 \mathrm{~mm}$. El color del cuerpo es marrón claro.

Cabeza. Primer y segundo segmento de la antena amarillos. Arista plumosa, 4 ramas dorsales y 3 ventrales más la terminal bifurcada. Con una cerda oral prominente. Las cerdas orbitales media y posterior se encuentran cerca del borde ocular, mientras que la anterior está hacia la parte interna. Triángulo ocelar marrón, el triángulo frontal de color amarillo polinoso y el frontal vitta marrón. Placa orbital amarilla y en la base de las cerdas orbitales de color marrón. Carina prominente, amarilla y ligeramente 
surcada. Gena de color amarillo claro. Ojos rojo vinoso. Proboscis y palpo maxilar amarillos.

Tórax. Atigrado y polinoso. En el mesonoto las cerdas y los pelos emergen de puntos oscuros. Posee 6 hileras de pelos acrosticales delante de las dorsocentrales anteriores y entre las cerdas dorsocentrales anteriores hay 5 hileras; sin cerdas preescutelares. Escutelo ligeramente oscuro más claro y hacia los lados. Cerdas escutelares anteriores divergentes. El tamaño de la cerda esternopleural media es un medio de la anterior. Patas de color amarillo oscuro. Alas de 3,30 mm de largo y 1,56 $\mathrm{mm}$ de ancho, pigmentadas con mayor intensidad sobre las venas transversales y más clara sobre la segunda celda posterior. Índices alares: $\mathrm{c}=5,50 ; \mathrm{ac}=1,33 ; \mathrm{hb}=0,33$; $4 \mathrm{c}=0,50 ; 4 \mathrm{v}=1,66 ; 5 \mathrm{x}=1,10 ; \mathrm{M}=0,46$; Prox. $\mathrm{x}=0,75$.

Abdomen. Color marrón oscuro en las áreas laterales de cada tergito y áreas amarillas en la zona dorsal y con línea media dorsal del primer al quinto tergito.

Genitalia interna. Ápice del edeago quitinizado y triangular en vista lateral (Figs 22 - 24), presenta dos crestas largas, quitinizadas y aserradas a lo largo de la zona dorsal. En vista dorsal, la punta del ápice del edeago es bífida. Las paráfisis tienen forma circular y poseen una cerda en el extremo libre además de micropubescencia en la misma zona. El apodema es membranoso en la parte distal pero se vuelve quitinizada conforme se acerca al ápice del edeago. La rama ventral del apodema es triangular. Índice del edeago=0,93.

Genitalia externa. Cerci presenta cerdas solo en la parte inferior (Fig. 25). Surestilo triangular. En el lado derecho con 10 dientes primarios y nueve (7-10) cerdas marginales; en el lado izquierdo tiene 10 dientes primarios y ocho (7-10) cerdas marginales. En la parte externa de los dientes primarios del lado izquierdo tiene una fila de 7 microproyecciones.

Descripción de la hembra. Alotipo (proviene de isolínea). Morfología externa: similar al macho pero ligeramente más clara.

Genitalia. Ovipositor triangular y medianamente quitinizado (Fig. 26). Posee una cerda larga en el extremo, $10(10-11)$ cerdas marginales y 2 espinas. No tiene dientes discales. Espermateca muy quitinizada y claramente separada en dos partes (Fig. 27); la parte superior es circular y porosa; la inferior tiene la forma de una copa y es igualmente porosa. Índice de la espermateca $=0,88$.

Etimología. El nombre hace referencia a la Quebrada de Yanayuyu, Pichincha, Ecuador.

\section{DISCUSIÓN}

Dentro del grupo D. mesophragmatica existen 17 especies, algunas de tamaño pequeño como $D$. mesophragmatica que mide de 2,9 a 3,6 mm (VILELA \& BACHLI, 1990) y especies grandes como D. amaguana de $6,5 \mathrm{~mm}, D$. shyri de $6,8 \mathrm{~mm}$ (Vela \& Rafael, 2004) y $D$. cashapamba sp. nov. de $5 \mathrm{~mm}$. Así mismo, la coloración de las especies varían de oscuras (D. mesophragmatica) a más claras (D. gaucha y D. pavani). Además dos de las especies descritas en este estudio, $D$. chorlavi $\mathrm{sp}$. nov. y $D$. yanayuyu sp. nov. presentan el tórax atigrado. Esta característica, común en los miembros del grupo $D$. repleta (VIlela, 1983), D. annulimana y D. peruenesis (RATCov \& Vilela, 2007; Tosi et al., 2007), es reportada por primera vez en el grupo D. mesophragmatica.

Otro carácter importante en el análisis morfológico del grupo $D$. mesophragmatica es la dirección de las cerdas escutelares anteriores. Dentro del grupo las especies con cerdas escutelares anteriores convergentes son: $D$. viracochi, $D$. shyri y $D$. chorlavi sp. nov. Estas tres especies se supone que conforman el subgrupo $D$. viracochi. El resto de especies del grupo D. mesophragmatica presentan cerdas escutelares divergentes y conformarían los subgrupos D. mesophragmatica у D. gaucha (МотА et al., 2008). Sin embargo, las especies ecuatorianas del grupo $D$. mesophragmatica aún no han sido estudiadas a nivel molecular por lo que la afiliación de las diferentes especies a los diferentes subgrupos es aún preliminar.

$\mathrm{Al}$ analizar la morfología de los edeagos de las especies se puede diferenciar aquellas que son muy similares a D. mesophragmatica, tales como D. brncici, $D$. gasici, $D$. camaronensis, $D$. canescens, $D$. altiplanica, $D$. gaucha, D. pavani, D. orkui, D. viracochi y $D$. chorlavi sp. nov. Las otras como D. amaguana, D. shyri, $D$. ruminahui y $D$. cashapamba sp. nov. presentan el patrón básico de $D$. mesophragmatica pero con algunas diferencias. Así mismo, las especies D. rucux sp. nov. y D. yanayuyu sp. nov. muestran notables diferencias.

Los análisis morfologicos de la genitalia, principalmente del edeago, de las cuatro especies nuevas indican que $D$. chorlavi sp. nov. y D. cashapamba sp. nov. están relacionadas con el grupo D. mesophragmatica; mientras que $D$. rucux sp. nov. y D. yanayuyu sp. nov. podrían ser consideradas como formas aberrantes del grupo. Estas discordancias se podrán aclarar con estudios citológicos y moleculares.

En el Ecuador se han encontrado nueve especies pertenecientes al grupo mesophragmatica y es muy posible que este número aumente. La formación de los Andes ha contribuido a la existencia de una gran variedad de ecosistemas que aún no han sido explorados (RAFAEL, 1992).

Agradecimientos. A la Pontificia Universidad Católica del Ecuador por financiar los proyectos: "Caracterización de nuevas especies ecuatorianas de Drosophila" No E29050 y "Diversidad del género Drosophila en bosques de Polylepis de la Provincia de Pichincha" No E29181. Al Dr. Carlos Ribeiro Vilela (Universidad de São Paulo, Brasil), por sus valiosos comentarios. A la Dra. Laura Arcos Decana de la Facultad de Ciencias Exactas y Naturales, a las Licenciadas Gabriela Pozo, Fernanda Salazar, Renata León, Andrea Pinos y Luna Figuero por su ayuda en el trabajo de campo y de laboratorio. Al Dr. Hugo Romero y Dr. Clifford Keil por los comentarios al presente trabajo. Finalmente, a la "Fundación Teleféricos de Pichincha", por permitir el ingreso a las instalaciones del Teleférico de Quito y a Idea Wild por la donación de los materiales. 


\section{REFERENCIAS}

Bächli, G.; Vilela, C. R.; Escher, S. A. \& Saura, A. 2004. The Drosophilidae (Diptera) of Fennoscadia and Denmark. Fauna Entomologica Scandinavica v.39. Leiden, Boston, Brill. 362p.

BraKe, I. \& BÄChLI, G. 2008. World catalogue of insects. Volume 9. Drosophilidae (Diptera). Stenstrup, Apollo Books. 412p.

Brncic, D. \& Koref-SAntibañez, S. 1957. The mesophragmatica species group of Drosophila. Evolution 11:300-310.

Mota, N. R.; Robe, L .J.; Vera, L. S.; Budnik, V. M. \& Loreto, E. 2008. Phylogeny of the Drosophila mesophragmatica group (Diptera, Drosophilidae): an example of Andean evolution. Zoological Science 25:526-532.

Nacrur, J. 1958. Genitalia masculina de Drosophila do grupo mesophragmatica (Diptera). Revista Brasileira de Biologia 18:243-249.

Rafael, V. 1992. Ecología y distribución del género Drosophila en el Ecuador. Revista Brasileira de Genética 15:213.

Rafael, V.; Arcos, G. \& Terán, A. L. 2000. Ecología y distribución del género Drosophila en Guayllabamba y El Quinche, provincia de Pichincha - Ecuador. Revista de la Pontificia Universidad Católica del Ecuador 65:130-155.

Ratcov, V. \& Vilela, C. R. 2007. A new neotropical species of spotthoraxed Drosophila (Diptera, Drosophilidae). Revista Brasileira de Entomologia 51(3):305-311.

Tosi, D.; Pereira, M. A. \& Vilela, C. R. 2007. Polytene chromosomes and phylogenetic relationships in ten Drosophila species of the annulimana group (Diptera, Drosophilidae). Genetics and Molecular Biology 39:223-230

Vela, D. \& Rafael, V. 2004. Three new andean species of Drosophila (Diptera, Drosophilidae) of the mesophragmatica group. Iheringia, Série Zoologia 94:295-299.

Vilela, C. R. 1983. A revision of the Drosophila repleta species group (Diptera, Drosophilidae). Revista Brasileira de Entomologia 27(1):1-114.

Vilela, C. R. \& Bächli, G. 1990. Taxonomic studies on Neotropical species of seven genera of Drosophilidae (Diptera). Bulletin of Entomological Society of America Suppl. 63:1-332. 